\title{
Interpretation of Hund's multiplicity rule for the carbon atom
}

\author{
Kenta Hongo $^{1}$, Ryo Maezono ${ }^{2}$, Yoshiyuki Kawazoe ${ }^{1}$, and Hiroshi Yasuhara ${ }^{1}$ \\ 1 Institute for Materials Research, Tohoku University, Sendai 980-8577, Japan and \\ 2 National Institute for Materials Science, \\ Sengen 1-2-1, Tsukuba 305-0047, Japan
}

\author{
M. D. Towler, and R. J. Needs \\ TCM Group, Cavendish Laboratory, \\ University of Cambridge, Madingley Road, \\ Cambridge CB3 OHE, United Kingdom
}

(Dated: November 27, 2018)

\begin{abstract}
Hund's multiplicity rule is investigated for the carbon atom using quantum Monte Carlo methods. Our calculations give an accurate account of electronic correlation and obey the virial theorem to high accuracy. This allows us to obtain accurate values for each of the energy terms and therefore to give a convincing explanation of the mechanism by which Hund's rule operates in carbon. We find that the energy gain in the triplet with respect to the singlet state is due to the greater electron-nucleus attraction in the higher spin state, in accordance with Hartree-Fock calculations and studies including correlation. The method used here can easily be extended to heavier atoms.
\end{abstract}




\section{INTRODUCTION}

From an analysis of atomic spectra, Hund found that the electronic configuration of the lowest energy is the one with the highest spin multiplicity $S$. Hund's multiplicity rule [1] explains almost all the configurations of the ground states of atoms, ions, and molecules and even their low-lying excited states in most cases. Even simple models such as frozen orbital Hartree-Fock (HF) theory generally adhere to the rule, although the mechanism by which Hund's rule is obeyed depends on the accuracy of the theoretical description. A widely accepted mechanism given originally by Slater 2] attributes it to a reduction in the electronelectron repulsion energy $V_{\text {ee }}$ in the highest $S$ state. This mechanism is in fact incorrect as it is based on the incorrect assumption of choosing the same orbitals for states with different $S$ (frozen orbitals). This assumption leaves the kinetic energy $T$ unchanged for different spin states, which cannot be correct as each eigenstate must satisfy the virial theorem. The virial theorem states that $2 T+V=0$, where $V$ is the total potential energy, which shows that if the potential energy is decreased the kinetic energy must increase. If the orbitals are allowed to relax self-consistently they are found to depend on $S$ states. 3, 4, 4, 5, 6, 1, 8, 9, 10, 11, 12, 13] The correct explanation of Hund's multiplicity rule is in fact that the higher spin state reduces the electron-nucleus interaction energy $V_{\mathrm{en}}$. Interestingly it turns out that states with larger $S$ have higher values of $V_{\text {ee }}$, in direct contradiction to the Slater mechanism.

Davidson 4] confirmed that Hund's multiplicity rule derives from a reduction in the electron-nucleus energy within the self-consistent HF approximation for the low-lying excited states of the helium isoelectronic series. The self-consistent HF method has been applied to explain Hund's multiplicity rule for all atoms (with the exception of $\operatorname{Zr}(Z=40)$ for which HF theory predicts an incorrect ground state) [3, 5, 6, 7, 8, 14], giving the same stabilizing mechanism. Boyd [9, 10] considered the same systems as Davidson but within a variational approach. He explained why larger values of $S$ give lower energies as follows. Larger $S$ implies more parallel spin pairs and hence a larger exclusion holes around each electron. Consequently, electrons can avoid each other more effectively and move closer to the nucleus, which reduces $V_{\text {en }}$. We refer to this as the less screening mechanism. We

should expect that when a mechanism lowers $V_{\text {en }}$ it should also increase $V_{\text {ee }}$, because the electrons will on average be closer to one another. The less screening mechanism is therefore in contradiction to Slater's argument which implies that $V_{\text {ee }}$ will be lower in the triplet state. 
Configuration interaction (CI) calculations have shown that the same mechanism operates in the low-lying excited states of small molecules such as $\mathrm{CH}_{2}, \mathrm{H}_{2} \mathrm{CO}$, and the first-row hydrides, LiH, BH, NH, and FH.[11, 12, 13]

The origin of Hund's multiplicity rule for heavier atoms has mostly been examined at the HF level. It is interesting to study the origin of Hund's multiplicity rule in heavier atoms beyond the HF level. However, the conventional approaches such as CI are difficult to apply for the following reasons: (1) The wave function must be constructed carefully from the configuration state functions (CSFs) giving the largest contributions to the correlation energy, which becomes a cumbersome task for heavier atoms. (2) A huge number of CSFs are required to give a sufficiently good description of the correlation, particularly the electronelectron cusps. In this paper we report a promising approach to this problem applied to the carbon atom as a prototype using quantum Monte Carlo (QMC) approaches such as the variational Monte Carlo (VMC) and more accurate diffusion Monte Carlo (DMC) methods.

\section{DESCRIPTION OF THE CALCULATIONS}

In $\mathrm{VMC}$, expectation values are calculated using an approximate many-body wave function, the integrals being performed by a Monte Carlo method. The approximate wave function normally contains a number of variable parameters, whose values are obtained by an optimization procedure.

$\mathrm{DMC}$ is a stochastic projector method for solving the imaginary-time many-body Schrödinger equation. 15] Exact imaginary-time evolution would in principle give the exact ground-state energy, provided the initial state has a non-zero overlap with the exact ground state. However, the stochastic evolution is not exact and the symmetry of the final state would not be fermionic, but bosonic. To maintain the correct fermionic symmetry the fixed-node approximation [16, 17] is used in which the nodal surface of the wave function is constrained to equal that of an approximate guiding wave function. If the nodal surface of the guiding wave function is accurate, fixed-node DMC will give a very accurate energy. The fixed-node DMC energy is less than or equal to the variational energy calculated with the guiding wave function and greater than or equal to the exact energy.

We used a single-determinant Slater-Jastrow guiding wave function. [18] The singleparticle orbitals were obtained from HF calculations using the GAUSSIAN98 code 19] and 
a $6-311 \mathrm{G}++(3 \mathrm{df}, 2 \mathrm{pd})$ basis set. At a nucleus the exact HF orbitals have cusps such that the divergence in the potential energy is cancelled by an equal and opposite divergence in the kinetic energy. Orbitals expanded in a Gaussians basis set cannot have cusps and the sum of the contributions from the kinetic and electron-nucleus energies diverges at the nucleus. In practice one finds that this results in wild oscillations in the local energy when an electron approaches the nucleus, which can lead to numerical instabilities in DMC calculations. To solve this problem we make small corrections to the single particle orbitals close to the nuclei which impose the correct cusp behavior. [20]

Electron correlation is introduced by multiplying the determinant by a Jastrow function which depends explicitly on the inter-electronic distances and obeys the electron-electron cusp conditions. 22] Our Jastrow factors take the form [23]

$$
J(\mathbf{R})=-\sum_{i>j}\left[\frac{A}{r_{i j}}\left[1-\exp \left(-\frac{r_{i j}}{F}\right)\right] \exp \left(-\frac{r_{i j}^{2}}{L_{0}^{2}}\right)+S_{1}\left(r_{i j}\right)\right]-\sum_{i, I} S_{2}\left(r_{i I}\right),
$$

where the indices $i$ and $j$ denote electrons and $I$ denotes ions. $F$ is chosen so that the cusp conditions [22] are obeyed, i.e., $F_{\uparrow \uparrow}=\sqrt{2 A}$ and $F_{\uparrow \downarrow}=\sqrt{A}$, and $S_{1}$ and $S_{2}$ are cusp-less. $S_{1}$ and $S_{2}$ are expressed as polynomial expansions in the inter-particle distances. We used a total of 12 variable parameters in the Jastrow factor whose optimal values were obtained by minimizing the variance of the VMC energy. 24, 25] The wave functions obtained by this procedure were used as the guiding wave functions for our DMC calculations. We used a timestsep of 0.001 a.u. within the DMC calculations, which gives very small timestep errors. All the QMC calculations were performed using the CASINO code. 21]

Arguments about the mechanism by which Hund's rule operates centre on the values of the individual energy terms $T, V_{\text {ee }}$ and $V_{\text {en }}$. As illustrated by Slater's early treatment, results which do not obey the virial theorem to high accuracy can give a spurious interpretation of the rule. It is therefore necessary to calculate the energy terms using an accurate method, and in the case of a stochastic method such as DMC, to evaluate them to a high statistical precision. Because there is no "zero-variance property" for the individual energy terms it is necessary to perform long Monte Carlo runs, and in our calculations we have accumulated the results over 200,000 steps.

The DMC method generates the mixed distribution, $\Psi_{v} \Phi_{0}$, where $\Psi_{v}$ and $\Phi_{0}$ are the guiding (VMC) and DMC wave functions, respectively. Evaluating the expectation value of the Hamiltonian $\hat{H}$ (or any operator which commutes with it) with the mixed distribution 
gives an unbiased estimate of the energy, i.e.,

$$
\langle\hat{H}\rangle_{m}=\frac{\left\langle\Psi_{v}|\hat{H}| \Phi_{0}\right\rangle}{\left\langle\Psi_{v} \mid \Phi_{0}\right\rangle}=\frac{\left\langle\Phi_{0}|\hat{H}| \Phi_{0}\right\rangle}{\left\langle\Phi_{0} \mid \Phi_{0}\right\rangle}=\langle\hat{H}\rangle_{p},
$$

where the subscript $m$ denotes the mixed estimator and $p$ denotes the pure estimator. However, for expectation values of operators which do not commute with the Hamiltonian the mixed estimate is different from, and generally less accurate than, the pure estimate. An approximation to the pure estimate can be obtained by combining VMC and DMC results in an extrapolated estimation. 26] The linear extrapolated estimator is

$$
\langle\hat{O}\rangle_{l}=2\langle\hat{O}\rangle_{m}-\langle\hat{O}\rangle_{v}=\langle\hat{O}\rangle_{p}+\mathcal{O}\left(\Delta^{2}\right)
$$

where $\Delta=\Phi_{0}-\Psi_{v}$. Other extrapolated estimators such as the squared extrapolation,

$$
\langle\hat{O}\rangle_{s}=\frac{\langle\hat{O}\rangle_{m}^{2}}{\langle\hat{O}\rangle_{v}}=\langle\hat{O}\rangle_{p}+\mathcal{O}\left(\Delta^{2}\right)
$$

can also be used, which have different error terms. In this study we found that the linear and squared extrapolations gave almost identical results, and we will quote results only for the linear one.

\section{RESULTS AND DISCUSSION}

In Table I we give VMC and DMC expectation values for the different each energy terms and for the virial ratio for the lowest energy triplet and singlet states of the carbon atom. For comparison, the HF and CI values of Pauncz et al. [6, 14] are also given. The exact nonrelativistic energy of the ground state of the carbon atom has been estimated to be -37.8450 a.u. 27] We can use this value and the Hartree-Fock energy of -37.689 a.u. to compute the percentages of the correlation energy recovered. The CI calculations of Pauncz et al. [6, 14] recovered $45.6 \%$ of the correlation energy, while a more modern CI calculation [28] (which did not report the individual energy terms) recovered 61.5\%. Our DMC calculations recover $88.5 \%$ of the correlation energy.

For each of the VMC, DMC and extrapolated DMC calculations we obtain a virial ratio within statistical error bars of its correct value of $-V / T=2$. It is straightforward to show from the virial theorem that the exact kinetic energy should be larger than the HF one in an isolated system. This inequality is obeyed by each of our calculations but is disobeyed by 
the CI calculations of Lemberger and Pauncz, [14] whose calculations also show a significant error in the virial ratio. This indicates that the virial ratio provides a sensitive test of whether the relationships between the different energy components are correct.

Comparing the DMC and extrapolated DMC as results with the HF ones for a particular state we find

$$
\begin{aligned}
V_{\mathrm{ee}} & <V_{\mathrm{ee}}^{\mathrm{HF}} \\
V_{\mathrm{en}} & <V_{\mathrm{en}}^{\mathrm{HF}} \\
T & >T^{\mathrm{HF}} .
\end{aligned}
$$

The introduction of correlation reduces both the electron-electron and electron-nuclear potential energies at the expense of increasing the kinetic energy. This increase can be understood as a consequence of the uncertainty principle; the increased correlation allows the charge density to contract towards the nucleus, which lowers $V_{\text {en }}$. However, the more localized charge density leads to an increase in the kinetic energy. Such a mechanism is not well described by the DCI (doubly excited CI) wave functions employed in Pauncz's calculations because the single excitation terms which are excluded contribute to the modification of the charge density. The spurious decrease in the kinetic energy seen in Pauncz's results might be attributed to this. In the QMC approach, on the other hand, such a modification of the charge density can be described by the one-body term in the Jastrow function [29] even within single determinant calculations.

Comparing the DMC and extrapolated DMC results for the singlet and triplet states we find

$$
\begin{aligned}
& V_{\mathrm{ee}}^{S=1}>V_{\mathrm{ee}}^{S=0} \\
& V_{\mathrm{en}}^{S=1}<V_{\mathrm{en}}^{S=0} \\
& T^{S=1}>T^{S=0} .
\end{aligned}
$$

Both the electron-electron repulsion and kinetic energy are lower in the singlet state, and the stabilization of the triplet occurs via the larger reduction in the electron-nucleus energy, corresponding to a contraction of the electron density towards the nucleus. This contraction is promoted by the presence of more spin-parallel pairs in the triplet which enables the contraction to occur without a large increase in the electron-electron interaction energy. 
The higher values of $V_{\mathrm{ee}}$ and $T$ in the triplet state are a consequence of the contraction depending on the spin state, which cannot be described within Slater's frozen orbital model.

Our results, which include a high-level description of correlation and satisfy the virial theorem to high accuracy, support Boyd's less screening interpretation of Hund's multiplicity rule for the carbon atom. It would not be difficult to extend this study to heavier atoms as, for example, all-electron DMC calculations of Xe $(Z=54)$ have recently been performed. [20]

\section{ACKNOWLEDGMENTS}

Our calculations were performed using the facilities of the Center for Computational Materials Science (IMR; Institute for Materials Research, Tohoku University), the Center for Information Science of JAIST (Japan Advanced Institute of Science and Technology), Kansai Research Establishment (JAERI; Japan Atomic Energy Research Institute) and the Numerical Materials Simulator of the Computational Materials Science Center (NIMS; National Institute for Materials Science). The authors would like to thank Professor Teruo Matsuzawa (JAIST) for the generous provision of computational facilities. K.H and R.M. would like to thank Dr. Taizo Sasaki for useful conversations. 
[1] F. Hund, Z. Physik 33, 345 (1925); ibid., 34, 296 (1925); "Linienspektren und periodisches System der Elemente", (Springer-Verlag OHG, Berlin, 1927).

[2] J. C. Slater, Phys. Rev. 34, 1293 (1929).

[3] S. Fraga, J. Karwouski and K. M. S. Saxena, Handbook of Atomic Data (Elsevier, Amsterdam, 1976).

[4] E. R. Davidson, J. Chem. Phys. 41, 656 (1964); ibid. 42, 4199 (1965).

[5] J. Katriel, Theor. Chem. Acta 23, 309 (1972).

[6] J. Katriel and R. Pauncz, Adv. Quantum Chem. 10, 143 (1977).

[7] J. P. Colpa, A. J. Thakkar, V, H. Smith, Jr, and P. Randle, Mol. Phys. 29, 1861 (1975).

[8] T. Koga, H. Matsuyama, J. S. Dehesa, and A. J. Thakkar, J. Chem. Phys. 110, 5763 (1999).

[9] R. J. Boyd and C. A. Coulson, J. Phys. B 6, 782, (1973); ibid. 7, 1805 (1974).

[10] R. J. Boyd, Nature 310, 480 (1984).

[11] K. V. Darvesh and R. J. Boyd, J. Chem. Phys. 87, 5329, (1987), ibid.; 90, 5638 (1989).

[12] K. V. Darvesh, P. D. Fricker, and R. J. Boyd, J Phys. Chem. 94, 3480 (1990).

[13] K. V. Darvesh, P. D. Fricker, and R. J. Boyd, Chem. Phys. 157, 99 (1991).

[14] A. Lemberger and R. Pauncz, Acta Phys. Acad. Sci. Hung. 27, 169 (1969).

[15] W. M. C. Foulkes, L. Mitas, R. J. Needs, and G. Rajagopal, Rev. Mod. Phys. 73, 33 (2001).

[16] J. B. Anderson, J. Chem. Phys. 65, 4121 (1976).

[17] P. J. Reynolds, D. M. Ceperley, B. J. Alder, and W. A. Lester, Jr., J. Chem. Phys. 77, 5593 (1982).

[18] R. J. Jastrow, Phys. Rev. 98, 1479 (1955).

[19] M. J. Frisch, G. W. Trucks, H. B. Schlegel, G. E. Scuseria, M. A. Robb, J. R. Cheeseman, V. G. Zakrzewski, J. A. Montgomery, Jr., R. E. Stratmann, J. C. Burant, S. Dapprich, J. M. Millam, A. D. Daniels, K. N. Kudin, M. C. Strain, O. Farkas, J. Tomasi, V. Barone, M. Cossi, R. Cammi, B. Mennucci, C. Pomelli, C. Adamo, S. Clifford, J. Ochterski, G. A. Petersson, P. Y. Ayala, Q. Cui, K. Morokuma, P. Salvador, J. J. Dannenberg, D. K. Malick, A. D. Rabuck, K. Raghavachari, J. B. Foresman, J. Cioslowski, J. V. Ortiz, A. G. Baboul, B. B. Stefanov, G. Liu, A. Liashenko, P. Piskorz, I. Komaromi, R. Gomperts, R. L. Martin, D. J. Fox, T. Keith, M. A. Al-Laham, C. Y. Peng, A. Nanayakkara, M. Challacombe, P. M. W. Gill, B. Johnson, 
W. Chen, M. W. Wong, J. L. Andres, C. Gonzalez, M. Head-Gordon, E. S. Replogle, and J. A. Pople, GAUSSIAN 98, Revision A.11.1, (Gaussian Inc., Pittsburgh, PA) (2001).

[20] A. Ma, M. D. Towler, N. D. Drummond, and R. J. Needs, unpublished.

[21] R. J. Needs, M. D. Towler, N. D. Drummond, P. R. C. Kent, G. Rajagopal, and A. J. Williamson, CASINO version 1.5 User's Manual, University of Cambridge, Cambridge, 2002.

[22] T. Kato, Commun. Pure Appl. Math. 10, 151 (1957).

[23] A. J. Williamson, S. D. Kenny, G. Rajagopal, A. J. James, R. J. Needs, L. M. Fraser, W. M. C. Foulkes, and P. Maccallum, Phys. Rev. B 53, 9640 (1996).

[24] C. J. Umrigar, K. G. Wilson, and J. W. Wilkins, Phys. Rev. Lett. 60, 1719 (1988).

[25] P. R. C. Kent, R. J. Needs, and G. Rajagopal, Phys. Rev. B 59, 12344 (1999).

[26] D. M. Ceperley and M. H. Kalos. in Monte Carlo Methods in Statistical Physics, edited by K. Binder (Springer, Berlin, 1979), pp. 145-194.

[27] E. R. Davidson, S. A. Hagstrom, S. J. Chakravorty, V. Mesier Umar, and C. Froese Fischer, Phys. Rev. A 44, 7071 (1991).

[28] T. Noro, M. Sekiya, and T. Koga, Theor. Chem. Acc. 98, 25 (1997).

[29] See Figures 7 and 8 in reference [15]. 
TABLE I: Energies for the triplet and singlet states of the carbon atom. All energies are given in hartrees. Standard errors in the QMC results are indicated in parentheses.

\begin{tabular}{ccccccc}
\hline Method & State & Energy & $V_{\text {ee }}$ & $V_{\text {en }}$ & $T$ & Virial ratio \\
\hline \hline HF & Triplet & -37.689 & 12.760 & -88.137 & 37.689 & 2.000 \\
& Singlet & -37.631 & 12.728 & -87.992 & 37.632 & 2.000 \\
CI & Triplet & -37.760 & 12.563 & -87.956 & 37.635 & 2.003 \\
& Singlet & -37.703 & 12.535 & -87.821 & 37.583 & 2.003 \\
VMC & Triplet & $-37.7717(1)$ & $12.629(1)$ & $-88.174(13)$ & $37.773(12)$ & $2.000(1)$ \\
& Singlet & $-37.6896(1)$ & $12.553(1)$ & $-87.932(13)$ & $37.689(13)$ & $2.000(1)$ \\
DMC & Triplet & $-37.8267(4)$ & $12.583(3)$ & $-88.203(13)$ & $37.793(12)$ & $2.001(1)$ \\
(mixed estimator) & Singlet & $-37.7623(6)$ & $12.509(5)$ & $-87.997(22)$ & $37.726(19)$ & $2.001(1)$ \\
DMC & Triplet & & $12.538(6)$ & $-88.231(29)$ & $37.811(26)$ & $2.002(2)$ \\
(linear estimator) & Singlet & & $12.464(9)$ & $-88.062(45)$ & $37.763(41)$ & $2.002(2)$ \\
\hline
\end{tabular}

${ }^{\mathrm{a}}$ Reference [6] ${ }^{\mathrm{b}}$ Reference [14] 